\title{
ARQUITECTURA Y ANATOMÍA FOLIAR DE Chrysophyllum cainito L. Y COMPARACIÓN CON OTRAS ESPECIES DE LA FAMILIA SAPOTACEAE
}

\author{
Ricardo Mar-Jiménez ${ }^{1}$ y Georgina Vargas-Simón ${ }^{1}$
}

\begin{abstract}
RESUMEN
Chrysophyllum cainito es un árbol frutal originario de Panamá y del que, a pesar de su importancia alimenticia y medicinal, existen pocos trabajos sobre su anatomía foliar. Se realizó una colecta de hojas en Cárdenas, Tabasco, México, y en el laboratorio se aplicaron las técnicas histológicas tradicionales para estudiar la arquitectura y anatomía foliar. En las muestras se identificó el patrón de venación y se determinó el grosor de la epidermis abaxial y adaxial, mesófilo, tipo y tamaño de los estomas, frecuencia estomática y se identificaron los tricomas, así como los tejidos de la nervadura central. La arquitectura foliar de esta especie es similar en algunas características a la de $C$. rufum y Manilkara spp. en cuanto a su venación broquidódroma como en la primera, y a sus venas intersecundarias y terciarias dispuestas al azar, como en la segunda. Se encontraron coincidencias en la forma rectangular de las células epidérmicas de este estudio con $C$. cainito nigeriano y $C$. rufum. Los estomas paracíticos son peculiares sólo en las hojas de C. cainito analizadas y en la especie tailandesa. La nervadura principal de $C$. cainito se considera bicolateral en un arco cerrado análogo con C. cainito tailandés. Así mismo, en este trabajo se identificaron coincidencias con las especies de la familia Sapotaceae a la que pertenece, como son la presencia de una capa epidérmica uniestratificada, hojas hipoestomáticas, cristales de oxalato de calcio, y tricomas tectores y ramificados (en "T") ubicados en la superficie abaxial. Se aportaron nuevos atributos tales como el espesor de la epidermis, tamaño de los estomas, frecuencia estomática y detalles de la nervadura central.
\end{abstract}

Palabras clave adicionales: Chrysophylloideae, estomas paracíticos, mesófilo, nervadura central, tricomas malpighiáceos

\begin{abstract}
Architecture and foliar anatomy of Chrysophyllum cainito $\mathrm{L}$.

Chrysophyllum cainito is a fruit tree native to Panama, but despite its nutritional and medicinal importance, there are few studies on its foliar anatomy. A collection of leaves was carried out in Cárdenas, Tabasco, Mexico, and in the laboratory, traditional histological techniques were applied to study the architecture and foliar anatomy. The venation pattern was identified and the thickness of the abaxial and adaxial epidermis, mesophyll, type and size of stomata, stomatal frequency, and trichomes were identified, as well as the tissues of the midrib. The foliar architecture of this species is similar in some characteristics to that of $C$. rufum and Manilkara spp. as the fact that they have brochidodromous venation, and present intersecond and tertiary veins randomly arranged. The rectangular shape of the epidermal cells in this study was found to coincide with Nigerian $C$. cainito and $C$. rufum. Paracytic stomata are peculiar only in the analyzed $C$. cainito leaves and in the Thai species. The main rib of $C$. cainito is considered bicolateral in a closed arch analogous to $C$. cainito Thai. Likewise, in this work, coincidences with the species of the Sapotaceae family to which it belongs were identified, such as the presence of a unilayered epidermal layer, hypostomatic leaves, calcium oxalate crystals, and tector and "T" branched trichomes located on the abaxial surface. New attributes such as epidermis thickness, stomatal size, stomatal frequency, and midrib details were added.
\end{abstract}

Additional keywords: Central rib, malpighian trichomes, mesophyll, paracytic stomata, Sapotaceae

\section{INTRODUCCIÓN}

Entre los frutos tropicales americanos se encuentra el caimito (Chrysophyllum cainito L.), un integrante de la familia Sapotaceae originario de Panamá, según el estudio de su biología molecular
(Petersen et al., 2012). Su centro de origen está asociado al bosque húmedo tropical. En México se distribuye en los estados cálidos de Morelos, Golfo de México y Península de Yucatán. Se encuentra ampliamente distribuido en Florida, en toda América tropical y en las islas del Caribe,

Recibido: Marzo 23, 2021

Aceptado: Octubre 25, 2021

${ }^{1}$ División Académica de Ciencias Biológicas. Universidad Juárez Autónoma de Tabasco. e-mail: ricardo_marj@hotmail.com; georgina.vargas@ujat.com (autora de correspondencia) 
ocasionalmente en Argentina y Perú (Morton, 1987), y adoptado en algunas áreas de Asia y África (Pasha y Uddin, 2019; Doan y Le, 2020; Prasawang y Srinual, 2020).

Son árboles muy frondosos con hojas alternas elípticas u oblongo-elípticas de 5-15 cm, márgenes lisos, base convexa y ápice acuminado, láminas cartáceas, lustrosas en el haz y marcadamente pubescentes en el envés. La pubescencia le otorga un color dorado a sus hojas, característica que lo hacen una especie favorita como ornamental.

Sus frutos pueden ser de color morado o verde, se consumen en fresco y se comercializan en pequeña escala (Álvarez et al., 2006; Castillo et al., 2014; Lema et al., 2017). Cada $100 \mathrm{~g}$ de fruto contiene 0,72-2,33 g de proteína, además de minerales (calcio y hierro) y vitaminas del complejo B y C, así como aminoácidos (Doan y Le, 2020). A la especie se le atribuyen propiedades medicinales ya que en sus hojas se han identificado alcaloides, compuestos fenólicos como flavonoides, esteroles y triterpenos, los cuales se utilizan para padecimientos de hipertensión y artritis reumatoide (Koffi et al., 2009; Doan y Le, 2020).

Los estudios anatómicos son una herramienta complementaria a la morfología y biología molecular y su aplicación se ha efectuado tanto a nivel botánico como en fisiología y ecología; mediante las técnicas histológicas se pueden demostrar modificaciones anatómicas de una especie como respuesta a diferentes ambientes (Crang et al., 2018). Siendo las hojas los órganos fotosintetizantes con alta actividad fisiológica y metabólica, la anatomía foliar se considera altamente valiosa.

Los estudios anatómicos foliares en Sapotaceae han sido notorios en especies como Diploon cuspidatum (Hoehne) Cronquist, Manilkara elata (Allemão ex Miq.) Monach., M. huberi (Ducke) Standl y Sideroxylon celastrinum (Kunth) T.D. Penn, donde se describen los tejidos comunes como el pecíolo, nervadura central, el mesófilo, tipos de tricomas, patrón de venación y estructuras secretoras (Monteiro et al., 2007; de Almeida et al., 2012; de Lima et al., 2019).

Dentro del género Chrysophyllum se efectuó una revisión anatómica foliar en C. rufum Mart., en la que se detectó la presencia de tricomas malpighiáceos, cristales de oxalato de calcio y laticíferos; se describieron los estomas, el mesófilo dorsiventral y el patrón de venación, cuyas características coinciden con las descritas para la familia Sapotaceae (Santos, 2019).

Con respecto a estudios anatómicos foliares con C. cainito, resalta el realizado en ejemplares de Tailandia (Prasawang y Srinual, 2020), donde las observaciones demostraron diferencias significativas de esta especie con C. roxburghii G. Don, respecto a la forma y contorno de las paredes de las células epidérmicas, presencia o ausencia de tricomas en forma de " $T$ ", presencia o ausencia de inclusiones y forma de los haces vasculares en la vena media. Con ejemplares de Nigeria se realizó una comparación anatómica foliar entre $C$. cainito, C. albidum G.Don y C. subnudum Baker, en la que se encontraron diferencias en la forma del pecíolo y de los haces vasculares (Inyama et al., 2016). Además, se caracterizó la cutícula y otros tejidos, como células epidérmicas del mesófilo y la vaina del haz vascular. Dado que son escasos los estudios realizados sobre esta especie, se realizó el presente este trabajo con el objetivo de aportar datos arquitectónicos y anatómicos de las hojas sobre ejemplares de una zona del trópico húmedo mexicano y su comparación con otras especies de la familia Sapotaceae.

\section{MATERIALES Y MÉTODOS}

Colecta de hojas. Se colectaron 20 hojas basales por árbol a partir de tres árboles de aproximadamente $12 \mathrm{~m}$ de alto, ubicados en un huerto familiar ubicado en la Ranchería Cucuyulapa, en Cárdenas, estado de Tabasco, México (17 $7^{\circ} 59^{\prime} 55^{\prime \prime} \mathrm{N} 93^{\circ} 14^{\prime} 47^{\prime}$ W). El clima es cálido-húmedo con abundantes lluvias en verano, con temperatura media anual de $26,2{ }^{\circ} \mathrm{C}$ y precipitación de 2000-2500 mm. El suelo es un Gleysol, de textura arcillosa (INEGI, 2020).

Arquitectura foliar. La determinación de la arquitectura foliar se realizó mediante aclaramiento con $\mathrm{NaOH}$ y tinción con safranina "O", de acuerdo a la técnica propuesta por Sandoval (2005), modificada. Para esto, las hojas se colocaron en cajas de Petri con $\mathrm{NaOH}$ al $25 \%$ durante 15 h para eliminar contenidos celulares. Posteriormente se eliminó el $\mathrm{NaOH}$ y se adicionó $\mathrm{NaClO}$ al $25 \%$ por $15 \mathrm{~h}$, luego se sustituyó la solución por $\mathrm{NaClO}$ al $50 \%$ por $30 \mathrm{~min}$, después de este tiempo, se lavaron repetidas veces con agua destilada para eliminar el exceso de cloro. Después las hojas se 
dejaron en safranina "O" durante 24 horas y pasado este tiempo se lavaron con agua destilada para eliminar el exceso del colorante. Por último, se fueron deshidratando gradualmente con etanol al $30,50,70$ y $96 \%$, con tiempos de 1 hora en cada cambio; una vez secas, se colocaron entre acetatos de vinilo para obtener material gráfico por medio de un escáner (Epson Perfection V300).

Las imágenes digitales sirvieron de base para la identificación del patrón de nervaduras, se determinó el tipo de vernación, de vena media principal, secundarias, las de posteriores órdenes y la vena última marginal basándose en diferentes autores (Ash et al., 1999; Stevens et al., 2001).

Anatomía foliar. Se efectúo el aclaramiento de hojas (Sandoval, 2005 modificado) para eliminar la cera de la epidermis adaxial y los tricomas en la epidermis abaxial: una parte de las hojas colectadas se seccionaron en porciones de $4 \mathrm{~cm}^{2}$ obtenidas de la zona media de la lámina (sin incluir a la nervadura central) y se sometieron a un remojo de 25 min en una solución de $\mathrm{NaOH}$ al $20 \%$ en cajas de Petri.

Las secciones se lavaron repetidas veces con agua destilada para eliminar el exceso de $\mathrm{NaOH}$, después se colocaron en una bandeja con papel absorbente para eliminar el exceso de agua y así poder realizar las impresiones con cianocrilato (Espinosa et al., 2002). Luego se procedió a medir la longitud y ancho de los estomas en un microscopio óptico Carl Zeiss con regla micrométrica, y la frecuencia estomática se obtuvo mediante un microscopio óptico Carl Zeiss con cámara clara.

La frecuencia estomática (FE) se obtuvo de acuerdo a la relación $\mathrm{FE}=$ Número de estomas entre la superficie foliar (Weyers y Meidner, 1990)

Para la identificación de los tricomas se realizaron cortes transversales a la lámina foliar en fresco con un micrótomo manual a $100 \mu \mathrm{m}$ y las secciones fueron teñidas con Floroglucinol- $\mathrm{HCl}$ al $1 \%$. La observación se realizó con un microscopio óptico Carl Zeiss con cámara fotográfica digital, y se caracterizó mediante imágenes digitales. Dicho proceso se realizó en el Laboratorio de Anatomía Vegetal del Colegio de Postgraduados (Montecillo, estado de México).

Otra porción de las hojas colectadas se fijó en FAA (Alcohol 96, $50 \mathrm{~mL}$; ácido acético glacial, $5 \mathrm{~mL}$; formaldehido $40 \%, 10 \mathrm{~mL}$; agua destilada, $35 \mathrm{~mL}$ ) para la caracterización del mesófilo; posteriormente, se sometieron al procedimiento histológico común de deshidratación con mezclas de alcohol etílico-xileno y su inclusión en parafina (Sandoval, 2005), luego se realizaron cortes transversales de $10 \mu \mathrm{m}$ de grosor con un micrótomo rotatorio. Las secciones fueron teñidas con verde fijo y rojo de aceite 7B, y montadas en bálsamo de Canadá (Sass, 1968) en el Laboratorio de Anatomía Vegetal del Colegio de Postgraduados. Estas preparaciones se utilizaron para medir el grosor de las epidermis adaxial y abaxial del mesófilo (tejidos en empalizada y esponjoso) y para la descripción de la nervadura principal. Las mediciones se hicieron en un microscopio Olympus con reglilla micrométrica.

\section{RESULTADOS Y DISCUSIÓN}

Arquitectura foliar. Las hojas de $C$. cainito son simétricas y presentan nerviación del tipo conduplicada. Se identifican por tener una vena media principal de tipo pinnada que va desde la base hasta el ápice, con venas secundarias del tipo broquidódroma, y otras intersecundarias marcadas muy débilmente en espacios uniformes, coincidiendo con la descripción hecha para esta especie por Stevens (2001); además, existen venas terciarias sinuosas del tipo reticulada ordenadas al azar, que además se reúnen de la misma forma con otras terciarias, formando ángulos. El curso de las terciarias es de tipo sinuoso ya que su curvatura cambia de dirección, pero orientadas hacia el margen de las hojas. Se presentan venas de cuarto y quinto orden del tipo dicotómico, muy finas; así mismo, se identificó una venación marginal extrema que se curva para formar "bucles", con aréolas pobremente definidas e irregulares (Figura 1).

Los patrones de venación coinciden con los de C. rufum (Santos, 2019), ambas broquidódromas; sus nervaduras secundarias son de menor grosor que la vena principal y esta especie tiene nervaduras secundarias de ocho a diez pares formando ángulos de $90^{\circ}$, aproximadamente, en el caso de $C$. cainito, aunque lo más frecuente son ángulos de $70^{\circ}$ y se llegan a contar hasta 36 pares, en promedio. La especie en estudio presenta venas intersecundarias y terciarias dispuestas al azar como en Manilkara spp. (M. elata y M. máxima) (de Almeida et al., 2012). Este tipo de particularidades son de carácter de diagnóstico para 
Sapotaceae y comunes en la subfamilia Chrysophylloideae, a la que pertenece esta especie, particularmente en la venación terciaria reticular (de Faria et al., 2017).
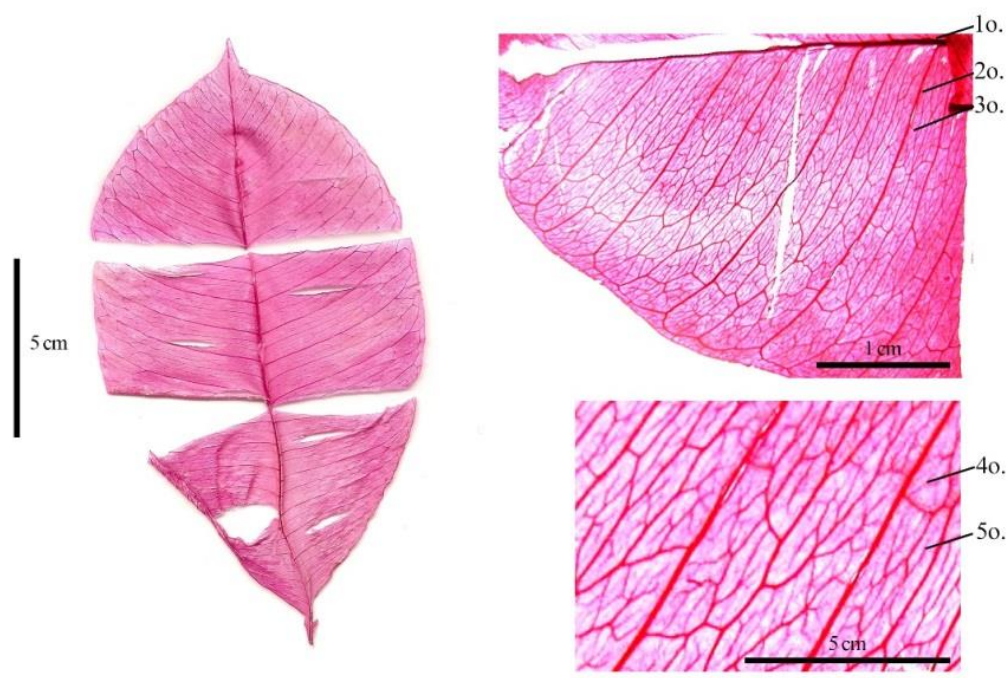

Figura 1. Patrones de nerviación de la hoja de Chrisophyllum cainito, izquierda hoja completa, derecha detalles de las nervaduras, primer hasta quinto orden

Al considerar su arquitectura, las nervaduras tienen una función de estabilización mecánica, y junto a la densidad de la hoja y el tejido celulósico, conforma elementos que le dan a $C$. cainito una clasificación media a la fractura de la lámina. Westbrook et al. (2011) la clasificó con un valor de $335,8 \mathrm{Jm}^{-2}$, por encima de Tabebuia guayacan (Seem.) Hemsl. (Bignoniaceae), con 164,8 $\mathrm{Jm}^{-2}$, y por debajo de Garcinia madruno (Kunth) Hammel (Clusiaceae), con 761,5 $\mathrm{Jm}^{-2}$. Se ha observado que el tipo de nerviación cerrada (broquidódroma) es común en especies de clima tropical, que tienen la particularidad de distribuir homogéneamente el agua, dirigiendo el flujo a los sitios donde pudieran existir mayores tasas de pérdida del líquido (Roth et al., 2001).

\section{Anatomía foliar}

- Superficie epidérmica. Las células de la epidermis en vista paradermal tienen paredes anticlinales ligeramente sinuosas en la superficie adaxial (Figura 2); en cambio las de la epidermis abaxial tienen paredes rectas, aunque por la abundancia de estomas no se distinguen claramente (Figura 3).

Tanto en este estudio como en ejemplares de $C$. cainito de Tailandia y otras especies de Sapotaceae, las células epidérmicas son caracterizadas por tener paredes rectas en la superficie adaxial y muy sinuosas en la abaxial (Prasawang y Srinual, 2020).
Sin embargo, se ha demostrado que las hojas muy expuestas al sol, como en los ambientes tropicales, las paredes epidérmicas anticlinales se endurecen más rápidamente y tienden a ser menos onduladas (Fontenelle et al., 1994); tal es el caso de C. cainito y de otras especies de la misma familia, como se muestra en el Cuadro 1.

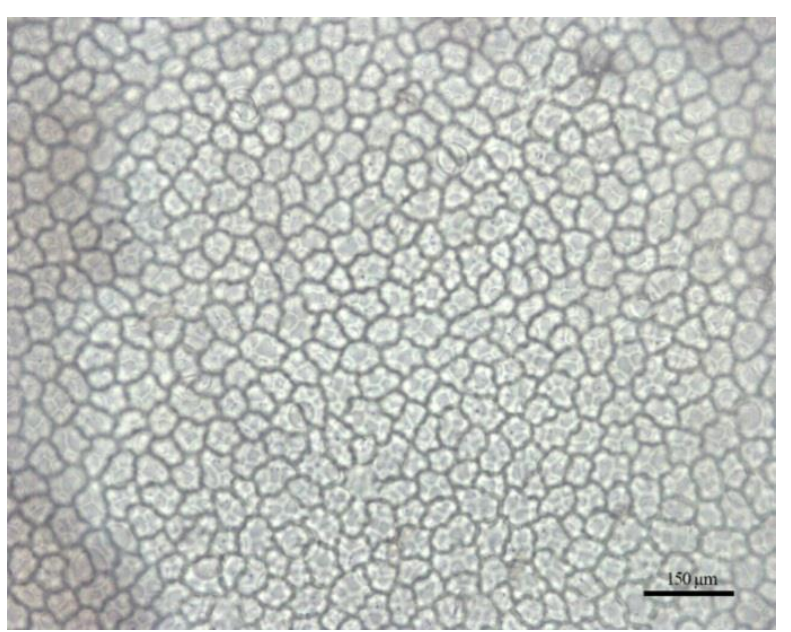

Figura 2. Vista paradermal en fresco de la superficie adaxial de la hoja de Chrysophyllum cainito mostrando células epidérmicas

La cutícula en la superficie abaxial es estriada (Figura 3) y en la adaxial es gruesa (Figura 4). La epidermis en ambas superficies es uniestratificada, 
sus células de forma rectangular y su grosor es doblemente mayor en la superficie adaxial, en

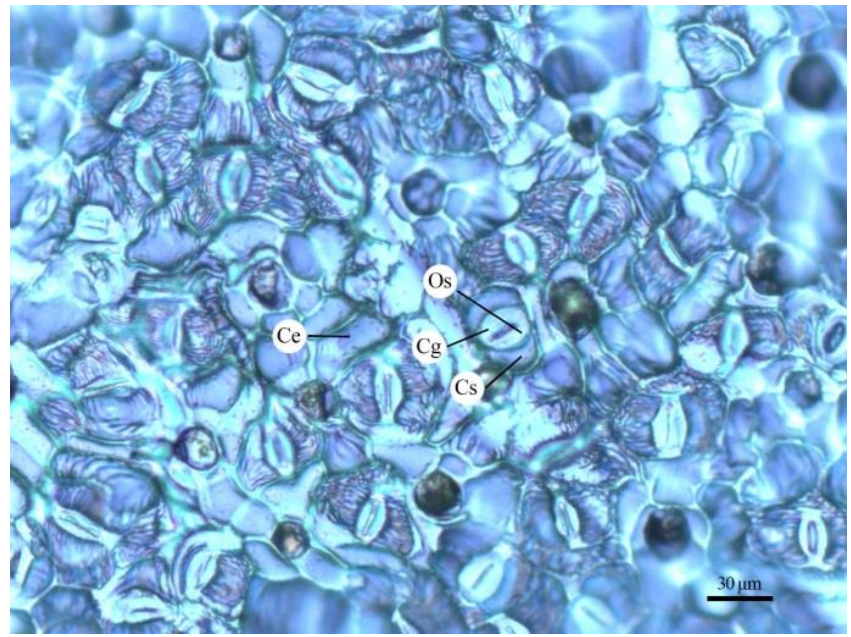

Figura 3. Superficie abaxial de la hoja de Chrysophyllum cainito, mostrando: células epidérmicas normales $(\mathrm{Ce})$, células guarda $(\mathrm{Cg})$, células subsidiarias (Cs) y el ostiolo (Os) de los estomas comparación con la abaxial (Figura 4; Cuadro 1), similar a lo referido por Prasawang y Srinual (2020).

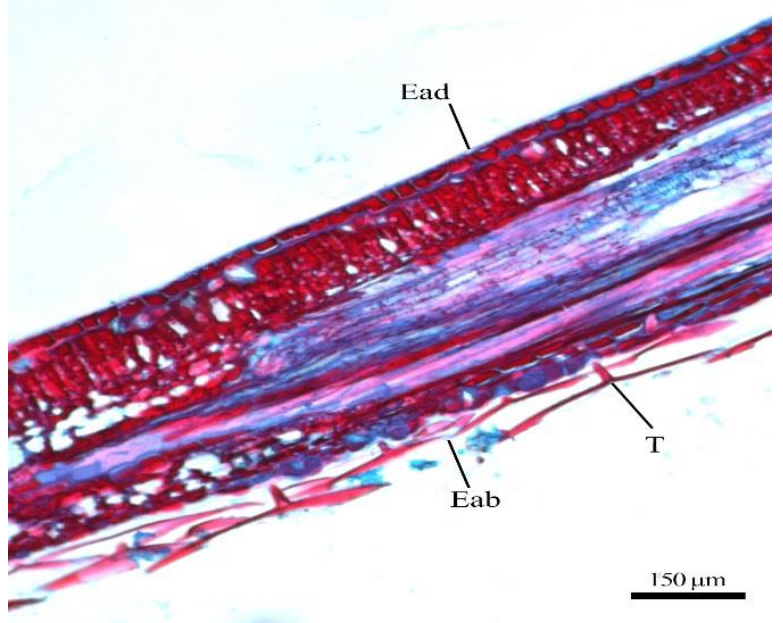

Figura 4. Corte transversal de la hoja de Chrysopyllum cainito, mostrando las epidermis adaxial (Ead) y abaxial (Eab), así como los tricomas $(\mathrm{T})$

Cuadro 1. Características anatómicas de las hojas de Chrysophyllum cainito

\begin{tabular}{|c|c|c|c|c|c|c|}
\hline Tejido & $\begin{array}{l}\text { Células epidérmicas } \\
\text { en superficie adaxial }\end{array}$ & $\begin{array}{l}\text { Células epidérmicas } \\
\text { en superficie abaxial }\end{array}$ & $\begin{array}{l}\text { Número de } \\
\text { estratos }\end{array}$ & $\begin{array}{c}\text { Forma de } \\
\text { células } \\
\text { epidérmicas }\end{array}$ & $\begin{array}{c}\mathrm{EE} \\
\text { adaxial } \\
(\mu \mathrm{m})\end{array}$ & $\begin{array}{c}\text { EE } \\
\text { abaxial } \\
(\mu \mathrm{m})\end{array}$ \\
\hline $\begin{array}{l}\text { Epidermis } \\
\text { (células } \\
\text { normales) } \\
\end{array}$ & $\begin{array}{l}\text { Paredes anticlinales } \\
\text { ligeramente sinuosas }\end{array}$ & $\begin{array}{l}\text { Paredes anticlinales } \\
\text { probablemente rectas }\end{array}$ & Uniestratificada & Rectangular & 20,61 & 11,76 \\
\hline Mesófilo & $\begin{array}{l}\text { Número de filas de } \\
\text { células en parénquima } \\
\text { en empalizada }\end{array}$ & $\begin{array}{l}\text { Número de filas de } \\
\text { células en parénquima } \\
\text { esponjoso }\end{array}$ & $\begin{array}{l}\text { Espesor del } \\
\text { parénquima en } \\
\text { empalizada }(\mu \mathrm{m})\end{array}$ & $\begin{array}{l}\text { Espesor del } \\
\text { parénquima } \\
\text { esponjoso }(\mu \mathrm{m})\end{array}$ & & \\
\hline & 1 & 5 & 84,6 & 76,5 & & \\
\hline
\end{tabular}

EE: espesor de la epidermis

La epidermis uniestratificada también se ha observado en otras especies de la familia Sapotaceae (Inyama et al., 2016; Duarte y Carneiro, 2016; de Lima et al., 2019). Sin embargo, en otras especies del género se han determinado epidermis multiestratificadas como en C. albidum G. Don y C. subnudum (Inyama et al., 2016). La forma rectangular de las células epidérmicas es similar a las de $C$. rufum, pero diferentes en $C$. cainito tailandés y a Diploon cuspidatum (Santos, 2019; Prasawang y Srinual, 2020).

Los valores medidos en el grosor de la epidermis de $C$. cainito son comparables a los de Pouteria beaurepairei (Glaz. y Raunk) Baehni para la epidermis adaxial y de $P$. venosa (Mart.) Baehni para la epidermis abaxial (Boeger et al., 2004). El grosor de la epidermis de la superficie adaxial generalmente es mayor en especies que crecen en climas tropicales, ya que es la superficie que está mayormente expuesta a la radiación solar, lo cual evita que el exceso de luz oxide a la clorofila que se encuentra en el mesófilo (Kume, 2017).

La variabilidad en el grosor de los tejidos foliares depende estrechamente de las condiciones ambientales (particularmente luz) en que se desarrollan las hojas (Fontenelle et al., 1994; Boeger et al., 2004; Crang et al., 2018).

- Estomas. Chrysophyllum cainito es una especie 
hipoestomática, con estomas paracíticos (tipo rubiáceos), es decir, dos células guardas y dos células subsidiarias dispuestas en forma paralela (Figura 3); su distribución es al azar, muy abundantes, lo que dificultó poder diferenciar las células epidérmicas para obtener el índice estomático en la superficie abaxial; las medidas de dichas células se muestran en el Cuadro 2, así como la frecuencia estomática. Las hojas hipoestomáticas están relacionadas a la mayor humedad relativa en la superficie abaxial y a la temperatura más alta en la superficie adaxial, lo cual se considera como una estrategia para evitar la entrada a organismos dañinos que generalmente se encuentran en el haz (Boeger et al., 2004; Crang et al., 2018).

Metcalfe y Chalk (1979) consideran a los estomas de tipo anisocítico más frecuentes en Sapotaceae y más raros el tipo paracítico (Cuadro 2); actualmente se ha identificado el tipo ciclocítico en $C$. roxburghii (Prasawang y Srinual, 2020), por lo que se denota una variabilidad en la familia botánica en dichas células.

La presencia de estomas pequeños y frecuentes en $C$. cainito se corresponde con su centro de origen, el cual está asociado al bosque húmedo tropical. De acuerdo con Drake et al. (2013) y Kardiman y Ræbild (2018), las condiciones de suelos húmedos favorecerían la formación de estomas pequeños y frecuentes mientras que la presencia de estomas grandes y escasos se corresponden con una mayor eficiencia en el uso del agua, pero tasas de fotosíntesis más reducidas.

Con relación a la frecuencia estomática, Ichie et al. (2016), describen una correlación negativa entre el tamaño de los estomas y su frecuencia, tal como se observó en nuestro estudio. Así mismo, las hojas provinieron de la zona basal del árbol, aunque se ha demostrado que la frecuencia puede variar entre hojas que ocupen distinta posición en la planta e incluso entre individuos de la misma especie cuando se desarrollan en ambientes diferentes (Nughes et al., 2013), y sobre todo en lo que respecta a la exposición a la radiación solar, lo cual explica el caso de Palaquium gutta (Sapotaceae), donde la frecuencia estomática es menor cuando el árbol se encuentra en condiciones de sombra que cuando crece en ambiente luminoso (Kardiman y Ræbild, 2018).

Cuadro 2. Características estomáticas en hojas de caimito Chrysophyllum cainito y de otras especies de la familia Sapotaceae. Promedios \pm SD

\begin{tabular}{|c|c|c|c|c|c|}
\hline Especie & Tipo & $\begin{array}{c}\text { Longitud } \\
(\mu \mathrm{m})\end{array}$ & $\begin{array}{l}\text { Ancho } \\
(\mu \mathrm{m})\end{array}$ & $\begin{array}{c}\text { Frecuencia } \\
\left(\text { estomas } \cdot \mathrm{mm}^{-2}\right)\end{array}$ & Fuente \\
\hline Chrysopyllum cainito & Paracíticos & $14,22 \pm 1,9$ & $7,57 \pm 0,9$ & $401 \pm 37,6$ & Este trabajo \\
\hline Chrysopyllum cainito & Paracíticos & - & - & - & $\begin{array}{l}\text { Prasawang y Srinual } \\
2020\end{array}$ \\
\hline Chrysophyllum rufum & Anisocíticos & 2,12 & 1,17 & $593 \pm 5,9$ & Santos, 2019 \\
\hline Diploon cuspidatum & Anisocíticos & - & - & - & de Lima et al., 2019 \\
\hline $\begin{array}{l}\text { Sideroxylon } \\
\text { celastrinum }\end{array}$ & Anisocíticos & 22,3 & 9,3 & 155,2 & Maiti et al., 2016 \\
\hline
\end{tabular}

- Tricomas. Los tricomas de C. cainito se encontraron distribuidos solamente en la superficie abaxial y en los bordes de la nervadura central. Fueron caracterizados como tectores, simples, no glandulares, con un pedúnculo corto y unicelulares ramificados de forma bífida (en "T"), clasificados como malpighiáceos, altamente lignificados y formando una capa densa de pubescencia (Figura 5). Esto coincide en su mayoría con las determinaciones para la familia que realizaron Metcalfe y Chalk (1979). Ocho de 13 especies de Manilkara también los presentan (de Almeida et al., 2012), particularmente $C$. cainito y $C$. roxburghii desarrolladas en Tailandia (Prasawang y Srinual, 2020), así como C. rufum (Santos, 2019).

El tricoma ramificado da una protección importante contra diferentes factores ambientales como altas temperaturas y fuertes emisiones de rayos UV, regulan la transpiración y sirven, además, como elementos para diferenciar géneros y especies (Ichie et al., 2016; de Faria et al 2017). Esta clase de tricomas se ha determinado en 38 familias de Dicotiledóneas y particulares en Malpighiaceae (Rao y Sarma, 1992). 
- Mesófilo. El mesófilo de la hoja de $C$. cainito es dorsiventral, contiene dos tipos de parénquima, en empalizada y esponjoso, cuyo espesor se aprecia en el Cuadro 3. El primero está constituido por una capa de células alargadas, colocadas en sentido perpendicular a la epidermis del haz. El esponjoso está constituido por cinco estratos, las células son de forma isodiamétrica con numerosos espacios intercelulares; en este tejido se observaron idioblastos con cristales prismáticos de oxalato de calcio (Figuras 6 y 7); en esta región no se distinguieron laticíferos.

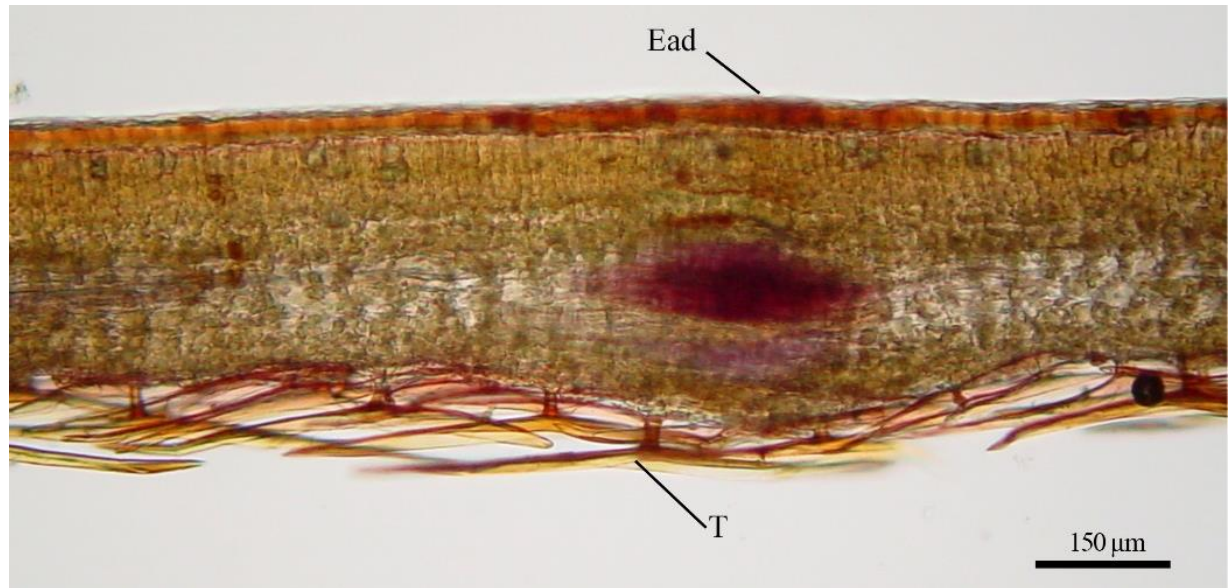

Figura 5. Corte transversal de la hoja de Chrysopyllum cainito, mostrando los tricomas malpighiaceos (T) en la superficie abaxial. Epidermis adaxial (Ead)

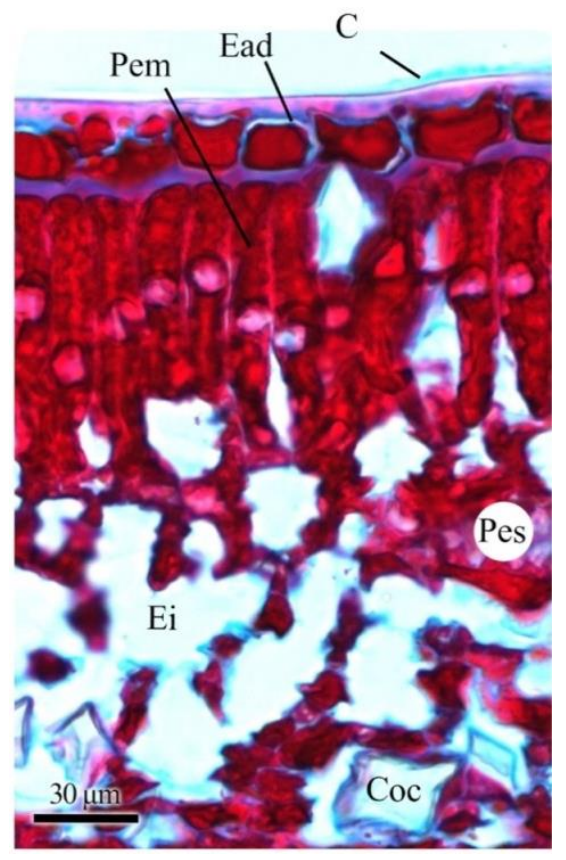

Figura 6. Corte transversal de la hoja de Chrysophyllum cainito, mostrando la cutícula (C), epidermis adaxial (Ead), parénquima en empalizada (Pem), parénquima esponjoso (Pes), espacio intercelular (Ei) y cristal de oxalato de calcio $(\mathrm{Coc})$

En este estudio las características del mesófilo se diferencian con las del $C$. cainito tailandés, el cual

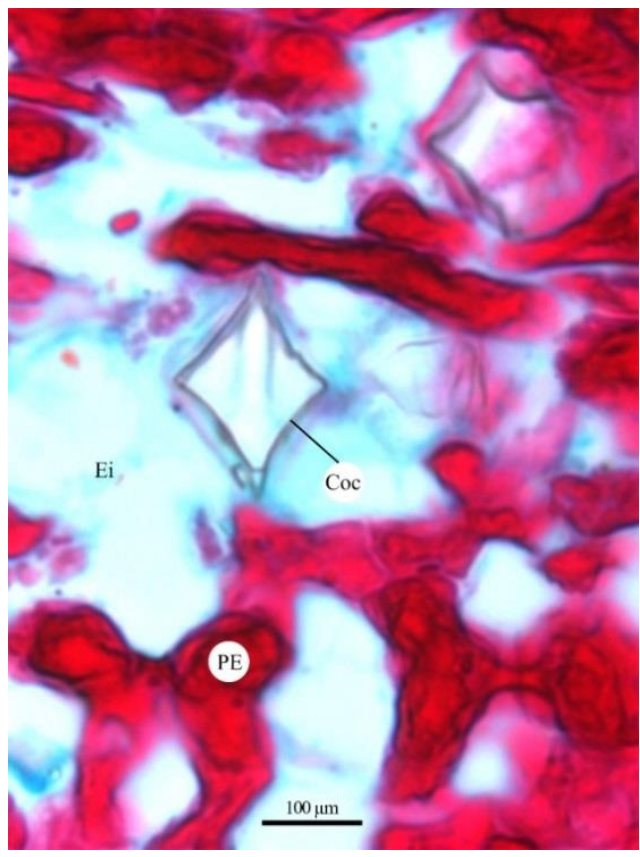

Figura 7. Detalle del cristal de oxalato de calcio en parénquima esponjoso (Pes) de la hoja de Chrysophyllum cainito. mostrando espacio intercelular (Ei) y cristal de oxalato de calcio (Coc)

mostró uno a tres estratos de parénquima en empalizada y diez del esponjoso (Prasawang y 
Srinual, 2020). Las células alargadas del tejido en empalizada en $C$. cainito, son equiparables en grosor con las del tejido esponjoso, en igual proporción, como en $M$. subsericea (Mart.) Dubard; sin embargo, en $P$. beaurepairei y $P$. venosa (Cuadro 3), el tejido de mayor espesor es el esponjoso (Boeger et al., 2004). Cuando las hojas tienen una sola capa de epidermis, parénquimas en empalizada y esponjosos bien desarrollados se consideran hojas mesomórficas como en este caso (Boeger et al., 2004).
Los cristales de oxalato de calcio en Sapotaceae, también se pueden encontrar en el mesófilo $(C$. roxburghii) y en la nervadura central como en $C$. rufum (Santos, 2019; Prasawang y Srinual, 2020).

- Nervadura central. El haz principal de la nervadura central de $C$. cainito se considera bicolateral, dispuesto en un arco cerrado. Prasawang y Srinual (2020) lo denominan "forma de corazón" e Inyama et al. (2016) lo definen "en forma de V".

Cuadro 3. Comparaciones de las características anatómicas foliares de Chrysophyllum cainito y algunas especies de la familia Sapotaceae. Valores medios del número de estratos de células en parénquima en empalizada y en el esponjoso, espesor del parénquima en empalizada (Eem), y espesor del parénquima esponjoso (Ees)

\begin{tabular}{lcccl}
\hline \multicolumn{1}{c}{ Especie } & $\begin{array}{c}\text { Número de estratos en } \\
\text { empalizada/esponjoso }\end{array}$ & $\begin{array}{c}\text { Eem } \\
(\mu \mathrm{m})\end{array}$ & $\begin{array}{c}\text { Ees } \\
(\mu \mathrm{m})\end{array}$ & \multicolumn{1}{c}{ Fuente } \\
\hline Chrysophyllum cainito & $1 / 5$ & 84,6 & 76,5 & Este trabajo \\
Chrysophyllum cainito & $2 / \mathrm{n} .0$. & - & - & Inyama et al., 2016 \\
Chrysophyllum cainito & $1-3 / 10$ & - & - & Prasawang y Srinual, 2020 \\
Chrysophyllum rufum & $2 / 8$ & - & - & Santos, 2019 \\
Diploon cuspidatum & $2 / 10$ & - & - & de Lima, 2019 \\
Manilkara subsericea & - & 125,4 & 127,4 & Boeger et al., 2004 \\
Pouteria beaurepairei & $1 / 8$ & 29,26 & 112,86 & Boeger et al., 2004 \\
Pouteria venosa & - & 20,9 & 87,78 & Boeger et al., 2004 \\
\hline
\end{tabular}

n.o.: número no observable

En la Figura 8 se observan cuatro haces vasculares: el principal de mayor tamaño, dos accesorios que se encuentran dispuestos lateralmente y el cuarto que se encuentra al extremo superior derecho y pertenece a una vena secundaria; en la superficie adaxial, se identifica la epidermis. En la parte central del haz vascular se observan los radios de xilema que contienen elementos de vaso muy desarrollados. El floema compuesto por elementos cribosos de menor tamaño. El haz presenta una vaina esclerenquimática perivascular, la rodean a su vez los laticíferos, posición asociada al floema, peculiar en Sapotaceae, los cuales para esta familia botánica son articulados (Monteiro et al., 2007); la vaina de esclerénquima da una consistencia compacta a la nervadura central (Westbrook et al., 2011).

Muchas de las características anatómicas identificadas en este trabajo coinciden con los descritos para la especie (Inyama et al., 2016; Prasawang y Srinual, 2020) y para $C$. rufum (Santos, 2019).

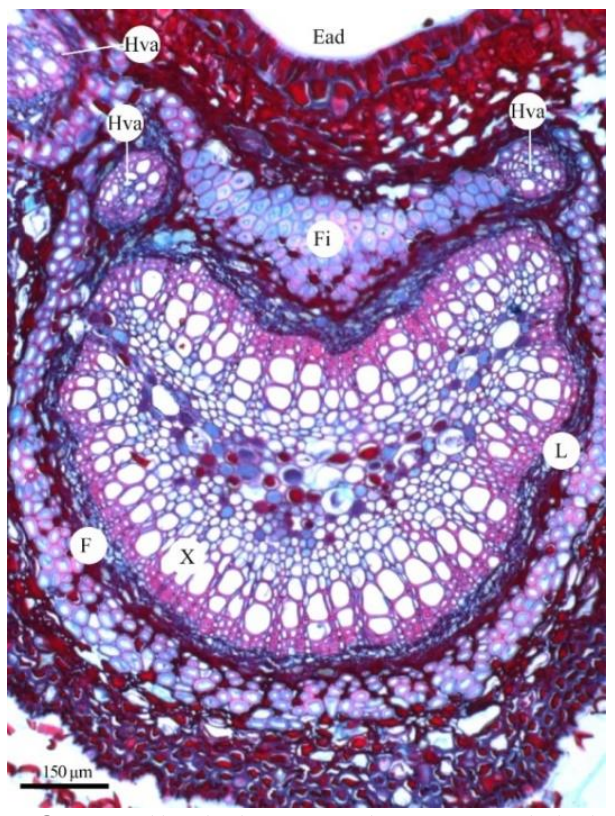

Figura 8. Detalle de la nervadura central de hoja de Chrysophyllum cainito en corte transversal, mostrando los haces vasculares accesorios (Hva), fibras (Fi), laticíferos (L), xilema (X), floema (F) y la epidermis adaxial (Ead) 


\section{CONCLUSIONES}

La arquitectura foliar de C. cainito es broquidódroma, resaltan las venas terciarias sinuosas del tipo reticulada y la venación marginal extrema curveada para formar "bucles". Las células de la epidermis en la superficie adaxial tienen paredes ligeramente sinuosas, pero en la superficie abaxial tienen paredes rectas. La epidermis es uniestratificada con células rectangulares, de un tamaño doblemente mayor en la superficie adaxial que en la abaxial. La primera está cubierta por una cutícula gruesa, en la abaxial no se distinguió. La hoja de $C$. cainito es hipoestomática con estomas paracíticos y una frecuencia estomática de 401,8 con una longitud de $14,2 \mu \mathrm{m}$ y ancho de $7,5 \mu \mathrm{m}$, en promedio.

Los tricomas se ubican exclusivamente en el envés de las hojas y en los bordes de la nervadura central, son del tipo tectores, unicelulares, de forma bífida y malpighiáceos, lignificados, que cubren considerablemente la superficie abaxial. $\mathrm{Su}$ mesófilo es dorsiventral, compuesto por parénquima en empalizada y esponjoso; el primero tiene solamente una capa de células alargadas que miden en promedio $84 \mu \mathrm{m}$, y el segundo con 76,5 $\mu \mathrm{m}$ y cinco hileras de células. Se identificaron cristales prismáticos de oxalato de calcio en esta zona.

En cuanto a la nervadura central, el haz vascular se caracteriza por ser bicolateral dispuesto en arco cerrado, con dos haces vasculares accesorios. Rodeando al haz vascular se identifica una vaina esclerenquimática perivascular y los laticíferos.

Se identificaron ciertas diferencias con ejemplares de la literatura, pero hay coincidencias con algunas especies de Sapotaceae y Chrysophylloideae a la que pertenece, tales como capa epidérmica uni-estratificada, hojas hipoestomáticas, cristales de oxalato de calcio, tricomas tectores y ramificados (en " $T$ ") ubicados en la superficie abaxial.

\section{AGRADECIMIENTO}

A la Dra. Hilda A. Zavaleta-Mancera y al Lab. Miguel Vega Zúñiga del Laboratorio de Anatomía e Histoquímica Vegetal del Colegio de Postgraduados, Campus Montecillo, por su valiosa asesoría y facilidades brindadas.

\section{LITERATURA CITADA}

1. Álvarez-Vargas, J.E., I. Alia-Tejacal, V. LópezMartínez, C.M. Acosta-Durán, M. AndradeRodríguez, M.T. Colinas-León, I. DelgadoEscobar y O. Villegas-Torres. 2006. Caracterización de frutos de caimito (Chrysophyllum cainito L.) en el estado de Morelos. Revista Chapingo Serie Horticultura 12(2): 217-221.

2. Ash, A.W., B. Ellis, L.J. Hickey, K.R. Johnson, P. Wilf y S.L. Wing. 1999. Manual of leaf architecture: morphological descriptions and categorization of dicotyledonous and net-veined monocotyledonous angiosperms. Smithsonian Institution, Washington.

3. Boeger, M.R., L.C. Alves y R.R. Negrelle. 2004. Leaf morphology of 89 tree species from a lowland tropical rain forest (Atlantic forest) in South Brazil. Brazilian Archives of Biology and Technology 47(6): 933-943.

4. Castillo C.G., C.H. Ávila-Bello, L. López-Mata y G.F. de León. 2014. Structure and tree diversity in traditional popoluca coffee agroecosystems in the Tuxtlas Biosphere Reserve, Mexico. Interciencia 39(9): 608-619.

5. Crang, R., S. Lyons-Sobaski y R. Wise. 2018. Plant Anatomy. Springer. New York.

6. de Almeida-Jr, E.B., J.S. Araújo, F.S. SantosFilho y C.S. Zickel. 2012. Leaf morphology and anatomy of Manilkara Adans (Sapotaceae) from northeastern Brazil. Plant Systematics and Evolution 299(1): 1-9.

7. de Faria, A.D., J.R. Pirani, J.E.L. Ribeiro, S. Nylinder, M.H. Terra-Araujo, P.P. Vieira y U. Swenson. 2017. Towards a natural classification of Sapotaceae subfamily Chrysophylloideae in the Neotropics. Botanical Journal of the Linnean Society 185(1): 27-55.

8. de Lima, R.G.V., L.F. Lima, A.C. Ferreira, J.S. Araújo y C.S. Zickel. 2019. Leaf morphoanatomy of Diploon Cronquist (Sapotaceae Juss.). Biota Neotropica 19(1): e20180600.

9. Doan, H.V. y T.P. Le. 2020. Chrysophyllum cainito: A Tropical Fruit with Multiple Health Benefits. Evidence-Based Complementary and Alternative Medicine. Hindawi, vol. 2020, article $7259267,9 \mathrm{p}$. 
10.Drake, P.L., R.H. Froend y P.J. Franks. 2013. Smaller, faster stomata: scaling of stomatal size, rate of response, and stomatal conductance. Journal of Experimental Botany 64(2): 495-505.

11.Duarte, A.F.T. y CE. Carneiro. 2016. Flora da Bahia: Manilkara Adans (Sapotaceae) considerações sobre a anatomia foliar. Anais Seminário de Iniciação Científica. Número 20. https://n9.cl/54fzh (consulta de enero 11, 2021).

12.Espinosa-Osornio, G., G. Vargas-Simón y M. Engleman. 2002. Contribución al estudio de la anatomía foliar del icaco (Chrysobalanus icaco L.). Bioagro 14(1): 29-36.

13.Ichie, T., Y. Inoue, N. Takahashi, K. Kamiya y T. Kenzo. 2016. Ecological distribution of leaf stomata and trichomes among tree species in a Malaysian lowland tropical rain forest. J. Plant Res. 129(4): 625-635.

14.INEGI. 2020. Prontuario de información geográfica municipal de los Estados Unidos Mexicanos (Cárdenas, Tabasco). Instituto Nacional de Geografía e Informática. Aguascalientes. https://n9.cl/sbry4 (consulta de enero 17, 2021).

15.Inyama, C.N., F.N. Mbagwu y C.M. Duru. 2016. Taxonomic relationship on some Chrysophyllum species based on anatomical studies. Medicinal Aromatic Plants 5(2): 1-5.

16.Kardiman, R. y A. Ræbild. 2018. Relationship between stomatal density, size and speed of opening in Sumatran rainforest species. Tree physiology 38(5): 696-705.

17.Koffi N., K.E. Amolkon, M.S. Tiébré, B. Kakja y G.N. Zirihi. 2009. Effect of aqueous extract of Chrysophyllum cainito leaves on the glycaemia of diabetic rabbits. African Journal of Pharmacy and Pharmacology 3(10): 501-506.

18.Kume, A. 2017. Importance of the green color, absorption gradient, and spectral absorption of chloroplasts for the radiative energy balance of leaves. Journal of Plant Research 130(3): 501514.

19.Lema, P., M.A. Pérez, M.T. Martínez y H. Navarro. 2017. Importancia y caracterización de frutales criollos en dos comunidades de Tuxpan, Veracruz. Revista Colombiana de Ciencias Hortícolas 11(2): 306-313.

20.Maiti, R., H.G. Rodríguez, P.C.R. Balboa, J.G.M. Moncivais, H.A.D. Tijerina, J.C.G. Díaz y A. Kumari. 2016. Leaf surface anatomy in some woody plants from northeastern Mexico. Pakistan Journal of Botany 48(5): 1825-1831.

21. Metcalfe, C. y L. Chalk. 1979. Anatomy of the Dicotyledons. Vol. 1. Oxford, Claredon Press. London.

22.Monteiro, M.H.D.A., R.H.P. Andreata y L.J. Neves. 2007. Estruturas secretoras em Sapotaceae. Pesquisas, Botânica 58(1): 253-262.

23.Morton, J.F. 1987. Fruits of warm climates. Julia F. Morton. Miami, FL.

24.Nughes, L., M. Colares, M. Hernández y A. Arambarri. 2013. Morfo-anatomía de las hojas de Celtis ehrenbergiana (Celtidaceae) desarrolladas bajo condiciones naturales de sol y sombra. Bonplandia 22(2): 159-170.

25.Pasha, M.K. y S.B. Uddin. 2019. Minor edible fruits of Bangladesh. Bangladesh Journal of Plant Taxonomy 26(2): 299-313.

26.Petersen, J.J., I.M. Parker y D. Potter. 2012. Origins and close relatives of a semidomesticated neotropical fruit tree: Chrysophyllum cainito (Sapotaceae). American Journal of Botany 99(3): 585-604.

27.Prasawang, S. y A. Srinual. 2020. Comparative leaf and wood anatomical characteristics of Chrysophyllum (Sapotaceae) relate to taxonomy of the species in Thailand. Biodiversitas Journal of Biological Diversity 21(4): 1578-1587.

28.Rao, S.R. y V. Sarma. 1992. Morphology of 2armed trichomes in relation to taxonomy: Malpighiales. Feddes Repertorium 103(7-8): 559-565.

29.Roth-Nebelsick, A., D. Uhl, V. Mosbrugger y H. Kerp. 2001. Evolution and function of leaf venation architecture: a review. Annals of Botany 87(5): 553-566.

30.Sandoval, Z.E. 2005. Técnicas Aplicadas al Estudio de la Anatomía Vegetal. Instituto de Biología. Universidad Nacional Autónoma de México. México.

31.Santos, R.C.S.L. 2019. Anatomia, histoquímica e arquitetura foliar de Chrysophyllum rufum Mart. (Sapotaceae) ocorrente no semiárido baiano. Dissertação em Recursos Genéticos Vegetais), Universidade Estadual de Feira de Santana (UEFS). 57 p.

32.Sass, J.E. 1968. Botanical Microtechnique. 
Iowa State University Press, Iowa.

33.Stevens, W.D., C.U. Ulloa, A. Pool y O.M. Montiel. 2001. Flora de Nicaragua. Monographs in Systematic Botany from the Missouri Botanical Garden. MSB 85, Tomo 1.

34.Westbrook, J.W., K. Kitajima, J.G. Burleigh, W.J. Kress, D.L. Erickson y S.J. Wright. 2011. What makes a leaf tough? Patterns of correlated evolution between leaf toughness traits and demographic rates among 197 shade-tolerant woody species in a neotropical forest. The American Naturalist 177(6): 800811.

35.Weyers, J.D. y H. Meidner. 1990. Methods in Stomatal Research. Longman Scientific \& Technical. London. 
